Anuario Latinoamericano Ciencias Políticas y Relaciones Internacionales vol. 1, 2014

p. $107-126$

\title{
Geopolítica del gas en Sudamérica
}

\author{
Ana Lía del Valle Guerrero \\ UNIVERSIDAD NACIONAL DEL SUR, \\ ARGENTINA \\ $\checkmark$ analiaguerrero06@yahoo.com.ar
}

\begin{abstract}
RESUMEN
El propósito de esta ponencia es analizar los cambios ocurridos en el sector energético sudamericano entre 2004 y 2011 - centrados en el recurso gas - donde se observan situaciones que avanzan desde un conflicto bilateral, a una cuestión regional con la posterior incorporación de actores globales históricamente ajenos a la región. Estos procesos de cambio son analizados mediante la aplicación de una metodología cualitativa que, a través del análisis de discurso que realiza la geopolítica crítica, permite percibir las complejas relaciones de poder que se establecen entre los distintos actores extraregionales de carácter global - como Rusia, Irán y Francia - con los principales actores regionales - Brasil, Venezuela y Bolivia - mediante una serie de alianzas no tradicionales que se originan como consecuencia de cambios en el escenario regional y mundial.
\end{abstract}

PALABRAS CLAVE: geopolitica, relaciones internacionales, energía, conflictos.

\section{SUMMARY}

The purpose of this paper is to explain by means of a geopolitical approach the present state and perspectives of the South American energy sector between 2004 and 2011 - focused on the use of gas. There are situations where conflicts move from bilateral problems to regional ones. Beginning in 2004 as a binational crisis centered in Argentina and Chile, it became a regional and global one after the active participation of France and Russia which determined large-scale changes. These processes of change are analyzed by applying a qualitative methodology that, through the analysis of speech of critical geopolitics allows to perceive the complex power relations that exist between the actors outside the region - such as Russia, Iran and France - with key regional players - Brazil, Venezuela and Bolivia, through a series of non-traditional partnerships that arise as a result of changes in regional and global scenario.

KEYWORDS: geopolitics, international relations, energy, conflict. 


\section{Introducción}

Cuando se habla de energía en esta primera década del siglo XXI, las referencias obligatorias continúan siendo el petróleo o el gas natural, pues ambas suman cerca del $60 \%$ de la matriz energética mundial. Esta demanda de energía a escala global y regional presenta dos características destacadas: por un lado, el crecimiento de la demanda es cada vez mayor y, por otro, la oferta se encuentra estancada y, en algunos casos, en declinación. Según un informe de 2007 del Departamento de Energía de EEUU se espera que la demanda mundial de energía primaria aumente - entre 2004 y 2030 - en un 57\%, lo que implica duplicar el consumo de energía en el breve lapso de 26 años. Para cubrir estas expectativas de consumo, la producción de petróleo debería aumentar un 42\%, el gas natural un 65\% (alcanzando una cifra de 163 billones de pies cúbicos o el equivalente a 29.000 millones de barriles de petróleo) y el carbón un 74\%. Estos aumentos en el plano teórico son casi imposibles de concretarse en la realidad, puesto que se observa una contracción continua de la oferta, salvo por esporádicos nuevos descubrimientos, como en el caso de Brasil, donde su extracción es difícil, requiere grandes inversiones y, además, tiempo para su puesta en producción. Sin embargo, debe tenerse en cuenta en el futuro el impacto que puede producir el uso de recursos no convencionales como shale gas, shale oil, tight gas y tight oil.

El presente trabajo se compone de tres partes. En la primera se realiza un abordaje global y por regiones, desde el punto de vista económico, de la situación del gas natural basándose en el último informe del BP, Statistical Review of World Energy, de junio de 2011. La segunda parte presenta una explicación de los cambios sufridos en la geopolítica desde diferentes enfoques. Por último, la tercera parte, como síntesis de las anteriores, se centra en el análisis y explicación de la geopolítica del gas sudamericano.

\section{La situación del gas natural en 2011}

\section{Abordaje global y por regiones, desde el punto de vista económico}

Para tener un panorama actualizado de la situación energética global, centrada en el gas natural, el BP Statistical Review of World Energy 2011 presenta algunas cifras que muestran la situación del recurso desde diferentes puntos de vista. En primer lugar, desde el punto de vista del consumo, se observa un crecimiento global del 7,4\% (el mayor incremento desde 1984). Estados Unidos presenta un record histórico del crecimiento de consumo del 5,6\% en el período 2009/2010, así como un incremento en el mismo período en Rusia $(6,3 \%)$, China (21,8\%) e India (21,5\%). Sin embargo, los mayores incrementos del consumo a nivel global se produjeron en América del Sur, encabezados 
por Perú con el 56\%, seguido por Chile con el 51\% y Brasil con el 33,8\% de incremento del consumo.

Desde el punto de vista de la producción, se produjo un crecimiento similar al del consumo de 7,3\% a escala global. Algunos hechos destacados en relación al crecimiento 2009/2010 son: el incremento del 30,7\% en la producción de Qatar y el mayor crecimiento a nivel global del 108,4\% en la producción de Perú. Por otra parte, también se observa, respecto a la producción global en 2010, que Estados Unidos (19,3\%) desplazó (por el uso del shale gas) del primer puesto en la producción a Rusia $(18,4 \%)$ y el importante declive de la producción de Canadá (5\%) por cuarto año consecutivo. Además, se observa un aumento del $10 \%$ en cuanto a los intercambios comerciales, con un incremento del 22,6\% en los envíos de Gas Natural Licuado (GNL). Se destacan, en particular, los envíos qataríes de GNL con un crecimiento del 53,2\% y el aumento de las exportaciones rusas a través de gasoductos.

Desde el punto de vista histórico y teniendo en cuenta las variaciones producidas en el período 1980-2010, existe una tendencia declinante en Oriente Medio, más allá de ser la mayor región productora y de poseer la mayor relación reservas/producción. En general, las reservas de las demás regiones no muestran grandes variaciones en su producción dando un horizonte global de reservas de 58,6 años.

Se debe destacar que los países que ocupan los 3 primeros lugares a escala mundial en cuanto a reservas probadas - Rusia (23,9\%), Irán $(15,8 \%)$ y Qatar $(13,5 \%)$ - concentran el 53,2\% de las reservas mundiales de gas natural y, si sumamos los 15 primeros puestos, totalizan el $84,5 \%$ de las reservas. En este contexto, la región sudamericana representa sólo el $4 \%$ de las reservas mundiales, con un ratio producción/reservas de 45,9 años.

A nivel regional y analizando particularmente la situación en la región sudamericana, se observa un estancamiento en el consumo de Argentina, pero se debe recordar que el consumo interno en Argentina es, desde la década de los 70, el más alto de sur y centro América e inclusive el segundo más elevado a nivel mundial donde representa el 1,4\% del consumo mundial, con lo cual no tiene un gran margen para crecer. Asimismo, es llamativo el crecimiento del consumo de gas natural en el período 2009/2010 en varios países de la región, por ejemplo: en Perú (56,0\%), Chile (51\%) y Brasil (33,8\%).

La situación regional, en cuánto a reservas probadas, muestra que en la relación reservas/producción aparece, como más favorecido, Perú con reservas para 48,8 años, seguido por Brasil $(28,9)$ y Bolivia $(19,5)$. Cabe aclarar que no están disponibles los datos para Venezuela, la cual posee las mayores reservas de la región aunque aún no las explota y las utiliza para reinyectarla en la producción de petróleo. Con respecto a Argentina, sus reservas probadas estarían consideradas en 8,6 años, aunque otros estudios consideran una cifra menor, de sólo 6 años. Con respecto a la producción, se destaca el cambio en la relación 2009/2010 para Perú, con un crecimiento del 108,4\%, seguido por Brasil con un $23 \%$ y Bolivia con un 16,8\%, mientras que Argentina muestra el
Geopolítica del gas en Sudamérica

Ana Lía del Valle Guerrero 
principal descenso, con un 3,0\%, a pesar de seguir representando el 1,3\% de la producción mundial.

Por último, se puede realizar un análisis global basándose en los datos aportados por el informe de BP (2011), para conocer la situación actual por región, teniendo en cuenta los siguientes parámetros para el año 2010: reservas probadas, producción, consumo y ratio reservas/producción.

En primer lugar, se destaca que la región más favorecida, considerando todos los parámetros, es Oriente Medio con un horizonte de reservas del $40,5 \%$ del total mundial, un consumo $(11,5 \%)$ por debajo de la producción $(14,4 \%)$ y un ratio reservas/producción mayor a 100 años. En esta región se encuentran países que ocupan los primeros puestos en cuanto a reservas: Irán (2), Qatar (3), Arabia Saudita (4). Entre los 3 países suman casi el 63\% de las reservas de la región. Esta distribución de las reservas muestra claramente dos fenómenos simultáneos, la concentración y la fragmentación. Por un lado, existe una concentración espacial de reservas probadas en un número reducido de países en la región de Medio Oriente, pero por otro lado, ello implica también una fragmentación espacial en la distribución de los recursos a nivel global, puesto que lo poseen determinados territorios. Asimismo, esta situación representa, a nivel global y regional, una asimetría entre aquellos países que poseen el recurso y aquellos que deben importarlo y por lo tanto, una competencia entre países productores y consumidores. Al ser el gas natural un hidrocarburo más amigable con el ambiente y de menor costo relativo respecto al petróleo, se espera que la demanda continúe en crecimiento en las próximas décadas como ya se observa al analizar las variaciones 2009-2010. Esto, a su vez, generará mayor competencia por el control de los mercados y las reservas, puesto que los Estados deben garantizar la seguridad energética a su población. Sin embargo, sólo podrán hacerlo aquellos países que dispongan de los recursos necesarios para cubrir su déficit energético comprándolo en el exterior, ya que, en general, no coinciden los principales productores con los mayores consumidores.

En este contexto global y de acuerdo con los datos analizados, se pueden vislumbrar algunos escenarios regionales en los que podrían surgir en el futuro más posibilidades de conflicto en cuanto a un abastecimiento fluido de recursos y, por lo tanto, problemas de seguridad energética. La tabla no. 1, elaborada a base de los datos aportados por el informe de BP (2011), permite extraer las siguientes conclusiones para cada una de las regiones.

Desde el punto de vista de escenarios de futuros conflictos se destaca, en primer lugar, la región de América del Norte con reservas probadas del $5,3 \%$ del total mundial (la segunda más baja), con producción y consumo dentro de los más elevados del mundo, $26 \%$ y 26,9\% respectivamente, y con el menor ratio reservas/producción de todas las regiones de sólo 12 años. Sin embargo, no deben olvidarse los importantes cambios en su situación a partir del uso de nuevas tecnologías de fractura hidráulica combinada con perforación horizontal que han permitido reducir los costos de extraer shale 
$g_{a s^{1}}$ de la roca madre. En esta región se encuentran Estados Unidos y Canadá que ocupan el 1 y el 3 puesto, respectivamente, en cuanto a la producción del recurso a nivel mundial (se debe recordar lo mencionado al inicio del trabajo en cuanto a una producción declinante de Canadá en los últimos 4 años como un problema adicional). Ambas situaciones reflejan que, a pesar de ser importantes productores, también son grandes consumidores, EEUU en el primer lugar y Canadá en el 6. Esta situación, sumada a poseer las menores reservas de todas las regiones, hace avizorar un futuro de creciente dependencia de la región de abastecimiento desde otras regiones, lo cual genera también vulnerabilidad desde el punto de vista de la seguridad energética, que puede ser evitada mediante el desarrollo de los recursos no convencionales.

\begin{tabular}{lcccc}
\hline \multicolumn{1}{c}{ Región } & $\begin{array}{c}\text { Reservas } \\
\text { probadas } \\
\text { en \% en 2010 }\end{array}$ & $\begin{array}{c}\text { Producción } \\
\text { en \% en 2010 }\end{array}$ & $\begin{array}{c}\text { Consumo } \\
\text { en \% en 2010 }\end{array}$ & $\begin{array}{c}\text { Ratio reservas/ } \\
\text { producción }\end{array}$ \\
\hline América del Norte & 5,3 & 26 & 26,9 & 12,0 \\
América del Sur & 4,0 & 5,0 & 4,7 & 45,9 \\
y Central & 33,7 & 32,6 & 35,8 & 60,9 \\
Europa y Eurasia & 40,5 & 14,4 & 11,5 & +100 \\
Oriente Medio & 7,9 & 6,5 & 3,3 & 70,5 \\
África & 8,7 & 15,4 & 17,9 & 32,8 \\
Asia Pacífico & & &
\end{tabular}

Fuente: Elaborado por Ana Lía Guerrero con base en datos de BP Statistical Review of World Energy, 2011.

La otra región que se puede observar con futuros problemas es la que corresponde a Asia Pacífico, ya que con el 8,7\% de las reservas mundiales presenta un consumo $(17,9 \%)$ por encima de la producción $(15,4 \%)$ junto a un ratio reservas/producción de 32,8 años, uno de los más bajos a nivel mundial. Un problema que complejiza más a esta situación es la tendencia a un crecimiento del consumo, puesto que en la región se encuentran dos de los países de mayor población del mundo y con economías en crecimiento continuo, como son China e India.

Desde el punto de vista de las reservas, se encuentran en esa región los países que ocupan los puestos del 12 al 15 a nivel mundial, respectivamente, Indonesia, Australia, China y Malasia. En cuanto a la producción, China ocu-

1 Es un gas que se encuentra enquistado dentro de bloques de rocas sedimentarias formadas a partir de materiales orgánicos, en reservorios de baja permeabilidad. Para su extracción, las empresas de Estados Unidos han liderado un método conocido como fracturación hidráulica. La tecnología usa agua, arena y químicos para fragmentar formaciones rocosas y poder acceder al gas.
Geopolítica del gas en Sudamérica

Ana Lía del Valle Guerrero

Tabla 1.

Comparación por regiones respecto a la escala global para el año 2010 
pa el 7 puesto e Indonesia el 9 y en lo referente al consumo, a nivel mundial, China ocupa el 4 el puesto, Japón el 5 e India el 8. Indudablemente y más allá de toda discusión, mencionar a Japón, China e India en la misma región ya implica mencionar a las principales potencias económicas y la necesidad de consumo será sin duda creciente, así como los problemas para asegurar un abastecimiento fluido.

La región de Europa y Eurasia se encuentra a nivel regional en un aparente equilibrio entre la producción $(32,6 \%)$ y el consumo $(35,8 \%)$, con un ratio reservas/producción por encima de los 60 años. Sin embargo, es remarcable el hecho de que toda la región depende del abastecimiento de un único gran proveedor, Rusia, y en menor medida, de Noruega, lo que genera vulnerabilidad y dependencia de la mayoría de los países europeos del gas ruso.

La región de África muestra un consumo (3,3\%) por debajo de su producción $(6,5 \%)$ y un alto ratio reservas/producción (70,5 años). La historia política y económica de la región plantea situaciones de mayor dependencia de sus colonizadores y explotación indiscriminada de sus recursos. Por lo tanto, la posibilidad de puesta en el mercado de la producción está limitada por situaciones previas de los Estados involucrados, pues estas cuestiones determinarán las decisiones políticas futuras, respecto al uso del gas, que irán más allá de cuestiones meramente económicas, sumadas a la inestabilidad política de la región.

Por último, analizando los datos de la región de América del Sur y Central se observa que es una de las de menor consumo $(4,7 \%)$ y producción $(5 \%)$, mientras que en el ratio reservas/producción ocupa el tercer lugar con 45,9 años. Cabe acotar, además, que la principal reserva de gas de la región que pertenece a Venezuela permanece sin explotar y que los nuevos descubrimientos de hidrocarburos de Brasil aún no entraron en producción. Por otra parte, Bolivia, el segundo país en reservas de la región, no se encuentra explotando todo su potencial por falta de inversiones en infraestructura y Perú comienza a hacerlo, como se observa en el crecimiento de su producción del 108,4\% en el período 2009/2010. Todo lo expuesto indica una capacidad de producción que aún no es utilizada a pleno en relación a sus reservas y consumo. A ello se suma, en el futuro, el uso de recursos no convencionales, como el shale gas, donde Argentina ocupa el tercer lugar a nivel mundial.

En conclusión, realizando una comparación a nivel global entre regiones, se puede concluir que, a pesar de que la región de América del Sur y Central presenta el menor porcentaje de reservas a nivel mundial con el $4 \%$ del total, es la que tiene mayor potencial de crecimiento de la producción, puesto que sus principales reservas aún no han sido explotadas y, además, presenta un bajo consumo y un ratio reservas/producción alto que la posiciona como una de las futuras áreas proveedoras del gas. Se suma a ello una mayor estabilidad política en relación con otras regiones proveedoras de recursos, como África y Medio Oriente.

En coincidencia con este punto de vista, que no es el aceptado mayormente, apareció en estos días en la revista "Foreign Policy" una opinión de 
Amy Myers Jaffe ("Nuevo Herald" 2011), directora del Foro de Energía del Instituto Baker en la Universidad Rice, titulada "Adiós OPEC" donde sostiene que la OPEC perderá gran parte de su poder en la década de 2020, porque "las Américas, no el Medio Oriente, serán para entonces la capital mundial de la energía”. Sostiene que este cambio se dará por factores tecnológicos y políticos (creciente inestabilidad regional, al igual que en África que genera caídas de la producción frente a una mayor estabilidad en América). Sin duda, esta situación no ha pasado desapercibida para las grandes potencias, como China y Estados Unidos, que ya han iniciado negociaciones con Brasil para el futuro uso de sus recursos y han aumentado su presencia en la región, así como también Irán, Francia, España y Rusia, entre otros.

Se argumenta que, teniendo en cuenta que para cualquier país es esencial garantizar el suministro copioso de energía, cualquier acto que pudiese reducir ese flujo supondría una amenaza automática a intereses vitales de seguridad nacional. Por ello se consideran al petróleo y al gas como recursos naturales estratégicos y asegurarse los proveedores es una prioridad de los Estados para garantizar su seguridad energética.

\section{La geopolítica y sus diferentes enfoques}

\section{Geopolítica - geopolítica crítica - posgeopolítica - nueva geopolítica de la energía}

Al punto de vista económico, propuesto en la primera parte del trabajo centrado en reservas, producción, consumo e intercambios comerciales, se suma, en esta segunda parte, el punto de vista geopolítico como otro modo de abordaje de la realidad. Para comprenderlo es necesario hacer una breve síntesis de los cambios producidos en el campo de estudio de la geopolítica y las causas que lo motivaron a fin de llegar a entender la geopolítica del gas sudamericano. En principio, una definición que contiene las características básicas de la geopolítica es la siguiente: "La geopolítica es la ciencia que estudia las mutuas relaciones, influencias y acciones entre el Estado y el espacio, a fin de aportar conocimientos o soluciones de carácter político” (Marini 1985: 44). Por su parte, "El espacio geopolítico es el área geográfica en cuyo seno actúan recíprocamente los factores geográficos y políticos que se desea estudiar o resolver" (Marini 1985: 45).

En síntesis, se puede decir que la geopolítica enfoca políticamente al espacio geográfico, a través de una relación armónica entre geografía y política. Basándose en el artículo desarrollado por Hutschenreuter (2008) acerca de la evolución del concepto de geopolítica se pueden extraer algunas ideas centrales: “...desde los años veinte, pero particularmente desde los años treinta, la geopolítica se transformó en una idea y una práctica de expansión territorial de base ultra-nacional, racial y militar (el nazismo) y quedó asociada a una
Geopolítica del gas en Sudamérica

Ana Lía del Valle Guerrero 
forma de ejercicio de poder estatal e interestatal que arrastró a las naciones al estado de guerra total. Seguidamente, el régimen bipolar implicó una granítica división geopolítica de las naciones en espacios o esferas de influencia..." (Hutschenreuter 2008: 2). No obstante, en los comienzos del nuevo orden internacional, la geopolítica inició un tímido retorno desde los años setenta y, en los ochenta, con el auge de los bloques regionales, regresó de la mano de la geoeconomía (Hutschenreuter 2008: 3).

En los 90, se destaca la geoeconomía centrada más en las cuestiones comerciales y económicas que en las políticas y territoriales. La geoeconomía establece relaciones entre geografía y economía a través de la importancia adquirida por el uso de los recursos naturales de un país mediante la acción política de los Estados. Tal es el caso de las grandes potencias que se disputan el dominio de las diferentes fuentes de energía, así como de aquellos minerales considerados críticos y de aplicación nuclear. La variedad, volumen y calidad de esos recursos naturales dan las primeras bases de apreciación del poder económico-político de una nación.

Posteriormente, la geopolítica también se vió afectada por la globalización caracterizada por el debilitamiento del factor territorial y con la desaparición de las fronteras nacionales, que la transformaron en una geopolítica sin territorio. Surge entonces una nueva geopolítica, la geopolítica crítica, que se centra en el poder político y deja de lado el factor geográfico. La geopolítica crítica se interesa por todo tipo de rivalidades sobre el territorio e intenta, a partir del análisis de discurso, observar de qué modo ingresa en las prácticas de actores políticos y públicos, tanto en escenarios pasados como presentes. Más que la investigación geopolítica tradicional enfocada en la geoestrategia en términos de planeación y resultados, la geopolítica crítica se dirige al examen de las ideas que les anteceden, sus circunstancias y su expresión en el plano del discurso estatal y provee una plataforma que pretende ser válida para todos los niveles del análisis espacial, no sólo de los asuntos internacionales, sino intrasociales en el marco de la glocalización (Barton 1997: 17-18 en Castro 2006: 191). En este contexto de cambio aparece la denominada posgeopolítica, caracterizada por las consecuencias de la aplicación del modelo económico neoliberal que permite penetrar en otros espacios a través de una desregulación de mercados que facilita la apertura de las economías y consigue los mismos resultados, pero sin el uso del poderío militar. Es, más bien, una relación entre economía y política, que con el territorio.

Por último, surge otro cambio relacionado con el enfoque centrado en un conflicto particular, pero de alcance mundial que relaciona un espacio geográfico particular que posee un recurso natural específico de ese territorio con las decisiones políticas de uso del recurso y que se conoce como la nueva geopolítica de la energía que propone ir más allá de una cuestión geoeconómica. Michael Klare (2003) sostiene que: “...en este mundo posterior a la guerra fría, las guerras por los recursos no son hechos fortuitos, ni inconexos, por el contrario: forman parte de un sistema geopolítico más amplio e interconectado. 
Las guerras del futuro se harán por la posesión y control de los recursos que precisan las modernas sociedades industriales para funcionar. Emerge entonces en éste marco, una nueva geografía de los conflictos, que ha trazado un mapa de una geografía estratégica donde no cuentan las fronteras políticas sino las concentraciones de los recursos".

En un libro posterior, (Klare 2008: 18) sostiene que “...El miedo mundial a la escasez energética se acompaña con una nueva geopolítica de la energía. Dentro del sistema internacional de poder que se va constituyendo podemos esperar que la lucha por la energía supere a todas las demás consideraciones, que los líderes internacionales lleguen a extremos para garantizar el suministro energético de sus países...El petróleo (y el gas natural) dejará de ser principalmente un lujo comercial, que se compra y se vende en el mercado internacional, para convertirse en un recurso estratégico preeminente en el mundo, cuya adquisición, producción y distribución absorberán cada vez más el tiempo, los esfuerzos y la atención de los gobiernos y los jefes militares”.

Otra componente a destacar es que, en esta nueva geopolítica de la energía se observa el resurgimiento de la presencia del Estado, y no tanto de las empresas y los intereses privados, en la búsqueda y dominio de los recursos energéticos que serían una de las dinámicas centrales de los asuntos mundiales en las próximas décadas. En el caso particular de Sudamérica, se muestra como un resurgimiento de los nacionalismos, tal cual lo muestran los procesos de nacionalización de los recursos en Bolivia, Venezuela y Ecuador.

De la combinación de todos estos cambios surge entonces la propuesta de analizar la geopolítica del gas sudamericano mediante un análisis geopolítico integral o integrador que recupere las contribuciones que las distintas tendencias fueron aportando a la disciplina. Por ello, se aborda el estudio reconociendo la existencia de componentes de la geopolítica clásica, por la presencia de Estados y sus decisiones políticas en un territorio, de la geoeconomía al poner énfasis en la presencia de un recurso (el gas natural) y sus usos a partir de decisiones políticas de los Estados y de la geopolítica crítica, porque el análisis de discurso de personalidades claves (Presidentes, Ministros de Economía, de Energía, de Defensa, etc.) deja traslucir ideas que no son expresadas en los acuerdos firmados entre los países de la región. Por último, desde la perspectiva de la nueva geopolítica de la energía se recupera el análisis de cada uno de los componentes existentes en el plano global y los posiciona en el contexto particular de la región sudamericana.

\section{Geopolítica del gas sudamericano}

Desde el punto de vista geopolítico y geostratégico adquieren relevancia entonces las relaciones entre los Estados involucrados y las decisiones políticas de uso del gas que se localiza en un determinado territorio y no en otros especificidad territorial del recurso - a lo que se suma su valor como recurso
Geopolítica del gas en Sudamérica

Ana Lía del Valle Guerrero 
natural estratégico, por el alto grado de incidencia en el desarrollo económico de un país. Coincidiendo con lo sostenido por Isbell “...hoy en día, la materia prima de la energía - particularmente petróleo y potencialmente también el gas natural - han surgido como una de las importantes variables en el contexto geopolítico mundial. Aunque América Latina posee relativamente pocos recursos energéticos, comparada con Medio Oriente o Rusia, en su propio contexto regional podría lograr una autosuficiencia energética, representar una fuente directa de oferta energética para EEUU y desempeñar un papel muy relevante en el juego geopolítico mundial de la energía" (Isbell 2008: 2).

Desde una perspectiva geopolítica moderna y según los conceptos que maneja la estrategia, las relaciones internacionales se centran en relaciones de poder entre los Estados que, desde el punto de vista estratégico, implican relaciones entre medios y fines, éstas a su vez generan relaciones de dependencia, donde el poder se percibe en función del grado en que dependen los fines de un actor de los medios del otro, estos últimos constituyen sus bases de poder (Delamer 2005:126). En síntesis, un país depende de otro cuando para su desarrollo debe someterse a las decisiones impuestas por el otro. Poseer los recursos o la tecnología otorga poder a quien lo posee y genera una relación de dependencia de aquellos países que poseen los recursos y quieren lograr sus fines, como autoabastecimiento energético, pero dependen de los medios (económicos, tecnológicos) que le aportan los actores globales extraregionales para conseguirlos. Por lo tanto, la vulnerabilidad que experimenta un país representa el grado de poder que llegan a tener otros países sobre él y que condiciona su accionar.

Esta situación es la que explica que, en el nuevo escenario geopolítico energético sudamericano, actores principales de peso global, como Irán, Francia, China (que poseen el recurso, la tecnología o los capitales necesarios para inversiones en infraestructura), aparecen junto a actores regionales, como Venezuela, Bolivia o Brasil. También se da la aparente contradicción entre alejarse de la órbita de EEUU para evitar la dependencia y la necesidad de acercarse a estos actores globales con los cuales se generan también relaciones de dependencia que buscan superarse a través de la transferencia de tecnología, que les permitiría en el futuro despegar por sí mismos y ampliar sus bases de poder, al contar con medios propios para alcanzar sus fines y llegar a ser dueños de sus recursos en el sentido más amplio. Tal es el caso de las relaciones Francia - Brasil, el cual pretende desarrollar submarinos nucleares para controlar sus nuevos descubrimientos en el presal.

Si se analiza la realidad latinoamericana desde una perspectiva energética, centrada en el gas, ésta se focaliza en los países sudamericanos que constituyen un espacio territorial caracterizado por condiciones contradictorias ya que, por un lado, cuentan con una gran variedad de recursos energéticos renovables y no renovables, que harían suponer la posibilidad de autosuficiencia energética a nivel regional, mientras que, por otra parte, la realidad muestra 
como varios países de la región presentan una situación de dependencia respecto de fuentes de abastecimiento externas localizadas a grandes distancias y, en otros casos, una vulnerabilidad energética que se hace más evidente a medida que avanzamos en el siglo XXI, producto del crecimiento de la demanda frente a una oferta estancada por falta de inversiones en infraestructura y en tareas de exploración.

El problema energético de mayor urgencia que enfrenta hoy la región sudamericana es el abastecimiento fluido de gas natural, predominante en la matriz energética regional que se agrava por la ausencia de políticas públicas de largo plazo en esta materia, o por la imprevisión histórica con que se ha procedido en algunos países. Tal es el caso de Argentina que se comportó como un país gasífero sin serlo, llevando a la sobreexplotación del recurso. Por ello, se hace evidente la necesidad de aplicar decisiones estratégicas para la región pensando en lograr la seguridad de abastecimiento energético que garantice el desarrollo y bienestar de las futuras generaciones, frente a una situación actual de vulnerabilidad y dependencia energética en la región.

Asimismo, desde una perspectiva geopolítica, el logro del autoabastecimiento energético de la región, en el mediano y largo plazo, es una necesidad que se hace cada vez más evidente si se observan los conflictos que surgieron entre los países de la región a partir del 2004, por ejemplo, Argentina-Chile, Bolivia-Argentina, Bolivia-Brasil, entre otros, y que llevaron a que la solución fuera buscar el abastecimiento desde el exterior de la región a través del uso del GNL y los barcos metaneros y regasificadores que comenzaron a surcar estas costas. Esta situación llevó a la región a tener una dependencia del suministro desde el exterior que genera una situación de vulnerabilidad energética en algunos casos, de dependencia en otros, o ambas situaciones simultáneamente, tal es el caso de Argentina.

En este contexto, surgen dos puntos de vista geopolíticos en la relación entre energía y seguridad. El primero, relacionado con la seguridad del Estado y la competencia internacional por asegurarse la posesión de recursos considerados estratégicos que otorgan poder al Estado. El segundo se ubica por encima de lo nacional, se preocupa por la seguridad, desarrollo y bienestar de la sociedad a nivel regional y global a través de procesos que favorezcan la integración energética. Más allá de lo deseable, la realidad muestra que prevalece el primer punto de vista (Cardozo 2006: 136-149).

En el caso sudamericano, en particular, los procesos de nacionalización de los recursos generan una territorialización de la política, producida como consecuencia de las decisiones y alianzas políticas entre los Estados - centradas no en el mercado, sino en el poder político que se ejerce sobre un territorio - que conducen a una dinámica territorial de incertidumbre e inestabilidad en materia energética a escala regional, la cual atenta contra el desarrollo regional y la mejora de la calidad de vida de la población involucrada en el proceso. Asimismo, en el escenario sudamericano se producen los tres mayores cambios en cuanto al crecimiento de reservas a nivel mundial. En primer término, en 2008,
Geopolítica del gas en Sudamérica

Ana Lía del Valle Guerrero 
grandes descubrimientos de hidrocarburos en Brasil, en el área del presal, que lo posicionan no sólo como un país que logró su autoabastecimiento energético, sino también como un país superavitario en recursos energéticos, que - sumado a su creciente presencia económica y política como país emergente con una de las mayores extensiones en superficie y numerosa población - agrega una mejora notable de su posicionamiento a nivel global. A ello se suma la última certificación de reservas de petróleo en Venezuela en 2010 (recordando que en muchos casos está asociado con yacimientos de gas) que lo colocan en primer lugar, por encima de Arabia Saudita. Por último, en 2011, el reconocimiento que las terceras reservas mundiales de shale gas se encuentran en Argentina.

Por su parte, también los principales países proveedores de recursos intentan maximizar los beneficios y ventajas que les da esta posición dentro del nuevo orden energético mundial, que algunos denominan "nacionalismo de recursos", que podría definirse como la administración de los flujos energéticos de acuerdo con los intereses vitales del Estado, intentando convertir ese poder latente en una ventaja política. Se siguen entonces los objetivos que disponen los respectivos gobiernos y en algunos casos se utilizan como instrumentos de política exterior (Klare 2008: 34-35). Estas empresas se involucran cada vez más en todos los eslabones de los circuitos de producción, transporte y comercialización de los productos, sea gas o petróleo.

El resurgimiento de conductas "estatistas" respecto a los recursos energéticos es consecuencia de la dependencia de los Estados de los hidrocarburos en un marco de escasez de la producción que genera amenazas a la seguridad energética y a la calidad de vida de la población de los países más desarrollados, a la vez que remarca condiciones de vulnerabilidad y dependencia en los principales países consumidores. Todas estas economías - tradicionales y emergentes - crecen y compiten por las mismas fuentes de energía.

Otra realidad que se debe enfrentar cuando se habla de la nueva geopolítica de la energía es que ésta seguirá dependiendo de los viejos recursos cada vez más escasos y con mayor demanda, puesto que a pesar de los intentos de científicos de todo el mundo por intentar sustituir estos recursos, la realidad es que ningún país consumidor de energía puede reemplazar en gran escala estos recursos. Al respecto, el informe del Departamento de Energía de EEUU de 2007 considera que estos combustibles continuarán satisfaciendo las necesidades energéticas mundiales en torno al 87\% aún en 2030 (DoE/EIA, IEO 2007, Tabla 2: 85).

Pero surge otra situación, la de aquellos países que poseen los recursos, pero no están en condiciones de defenderlos frente a la presión de los países ricos, pero escasos en recursos energéticos. Esta situación es clara en África. Sin embargo, en Sudamérica, frente a esta posibilidad, Brasil también avanza en su programa nuclear y desarrolla un submarino nuclear, con apoyo de Francia, a fin de proteger de agresiones externas sus recientes descubrimientos en el presal. Relacionada con esta situación y la posibilidad de firmar alianzas estratégicas con Estados que dispongan de tecnología, armas o conocimien- 
tos, surge la presencia en la región de actores de carácter global, como Rusia, Francia, Irán y China, entre otros. Frente a este cambio en la región, Estados Unidos reactiva la IV Flota para proteger sus intereses en la región con un poder al que ningún país latinoamericano puede oponerse. Esta competición intensificada por la energía considera que poseer energía es un factor que rivaliza con el poderío militar. Rusia es un claro ejemplo de esta situación. ${ }^{2}$

Paralelamente, otros países que poseen recursos, pero que tienen menor grado de apoyo, han adoptado otras medidas, por ejemplo, la propuesta de Venezuela y Bolivia de crear una "OPEC del gas natural" o alianzas estratégicas no tradicionales, como la realizada entre China y Rusia para disminuir el peso de Estados Unidos. Los expertos sugieren que cada nuevo barril que se añada a las reservas mundiales será más difícil y costoso de extraer que el anterior, estará a más profundidad en el subsuelo, más alejado de la costa, en entornos más peligrosos o en regiones del mundo más propensas al conflicto, más hostiles. Es probable que este mismo panorama se repita en la mayoría de los combustibles existentes, incluyendo el carbón, el gas natural y el uranio.

En la región, la situación geopolítica y estratégica que se presenta es la necesidad que tiene cada Estado de garantizar el abastecimiento fluido de energía a su población. Para ello requiere de la firma de acuerdos que permitan superar los conflictos de intereses que se presentan entre los actores de la región e internamente dentro de cada Estado, por ejemplo, en Argentina abastecer a su población o exportar a Chile, en Bolivia cumplir los contratos con Brasil o Argentina además de asegurar el abastecimiento interno. La mayoría de los países de América del Sur se encuentran frente al desafío de instrumentar políticas y estrategias que los pongan a salvo de posibles crisis energéticas. Ante este panorama, los distintos gobiernos optaron por realizar diferentes acciones:

Argentina realizó un complejo esquema de retenciones escalonadas a las exportaciones de hidrocarburos y derivados que generó una sobreexplotación de los recursos y falta de inversiones. A ello se suma el hecho de que el $90 \%$ de la matriz energética argentina depende del petróleo y del gas. Sin embargo, ambas producciones caen, casi de manera ininterrumpida, desde 1998. Probablemente sea uno de los países más afectados por la crisis energética el cual pasó de ser un país exportador a ser un país importador neto de energía, principalmente GNL. La otra apuesta es a la exploración y explotación de reservas de tight gas y shale gas descubiertas en un "megayacimiento" (Clarin 2011:2)

2 La posibilidad real de transformar al gas natural de un recurso estratégico (que podría ser utilizado como arma de dominación económica sobre otros países) a uno táctico, pudo observarse concretamente el 1 de enero de 2006, cuando el Presidente ruso Vladimir Putin puso en jaque al continente europeo. En uno de los inviernos más crudos de su historia, cortó el suministro de gas a Ucrania, principal distribuidor del recurso proveniente de Rusia, pero alejado políticamente de su ideología. Recordando las tácticas de la guerra fría, se puso en evidencia que el uso del gas representó un elemento de presión y poder - no militar sino económica - allí la idea de uso de un recurso natural como arma estratégica.
Geopolítica del gas en Sudamérica

Ana Lía del Valle Guerrero 
de gas no convencional en la cuenca neuquina, según anunció la Presidenta en diciembre de 2010, que permitiría ampliar las reservas de gas de 6 a 16 años. Sus principales problemas son inversiones en infraestructura, mayor precio y contaminación que genera.

En Chile, el 53\% de la matriz energética lo representa el sistema hidroeléctrico que se encuentra afectado por sequías que le impiden operar a pleno, sumado a una crisis de abastecimiento de gas que comenzó en 2004, cuando Argentina comenzó a restringir los envíos, lo que se acentúa aún más actualmente. La respuesta a la crisis ha sido el desarrollo de centrales termoeléctricas a carbón. Para 2015 se espera que esta tecnología represente casi un 30\% del principal sistema eléctrico del país (el triple de la actual participación). Otra estrategia ha sido la instalación de plantas regasificadoras que permiten traer el recurso desde cualquier lugar del mundo. El 13 de noviembre de 2009, se firmó en Chile el decreto que permite vender gas licuado de petróleo (GLP) a la Argentina y desde aquí a otros países como Uruguay, Paraguay y Brasil. La venta podría realizarse a partir de 2010 o 2011, cuando Argentina ajuste su marco legal. ${ }^{3}$ Argentina pasaría así de abastecedor a consumidor (Clarin 2009: 25).

Perú trata de cambiar su matriz energética dejando de utilizar los derivados del petróleo para aprovechar el gas natural de Camisea. La idea de un anillo energético que comunicara Perú con Chile y de allí a Argentina se dejó de lado y se reiniciaron contactos con Petrobras respecto a la instalación de refinerías en ese país. Sin embargo, Perú exportará este año 165 mil toneladas métricas de GNL y se proyectan realizar alrededor de 52 embarques de GNL a diversas partes del mundo (Tecnoil 2011). Además, la producción de gas natural ha pasado de 327 millones de pies cúbicos por día a alrededor de 700 millones, confirmando su mayor crecimiento (108\%) en la producción a nivel global entre 2009-2010.

Brasil es uno de los países menos afectados por el aumento del precio del petróleo, puesto que ya logró el autoabastecimiento petrolero y, además, con los nuevos descubrimientos de hidrocarburos será superavitario desde el punto de vista energético y tendrá mayor potencialidad para evitar crisis energéticas, propias y regionales, una vez que consiga liberarse de los inestables envíos de gas desde Bolivia. Posee una matriz energética con un 46,6\% de energía procedente de recursos renovables (la media es del 13\%). Sumado a ello, el uso de la caña de azúcar representa la segunda fuente de energía después del petróleo y este es otro recurso que lo aleja de la dependencia. El consumo de gas natural en 2010 aumentó el 35,5\%, en relación con 2009, con un promedio diario de 52,9 millones de metros cúbicos (Tecnoil 2011). Uno de los factores

3 El 27 de enero de 2011 se firmó este acuerdo y el ministro argentino de Vido sostuvo que "El objetivo es lograr un mayor intercambio. Es muy importante la integración, la simetría, en la que se va a ir trabajando. Se verá en determinado momento si a un país le sobra algo y el otro lo necesita para ver cómo se complementan. En este marco, se podría vender o comprar gas, depende de la situación. Por ejemplo, el intercambio que tenemos con Brasil funciona muy bien". 
para este crecimiento fue el uso más intenso de las plantas termoeléctricas que utilizan gas natural como combustible, ya que el aumento del consumo en ese sector fue del $171 \%$. Entre los consumidores habituales, el mayor incremento correspondió a la industria que elevó su consumo en un 20,15\%, mientras que el consumo residencial creció el 7,2\% y el comercial el 6,26\%.

Bolivia posee la segunda reserva de gas de la región, siendo su proveedor más importante. La inestabilidad política y la inseguridad jurídica le impidieron consolidar esa posición por falta de inversiones en infraestructura que le permitan explotar el recurso. Inclusive presenta conflictos en el abastecimiento interno, ya que la nacionalización de los hidrocarburos, por sí sola, no genera riqueza - son necesarias las inversiones extranjeras cuando el país no posee los recursos económicos para tornarlos rentables y a partir de allí generar ganancias que puedan distribuirse entre la población. Yacimientos Petrolíferos Fiscales Bolivianos (YPFB Corporación) invertirá este año más de 43,2 millones de dólares en tareas de exploración y explotación de hidrocarburos en la perspectiva de elevar en un 15\% la producción de petróleo, gas natural y otros líquidos asociados en el departamento de Cochabamba (Tecnoil 2011). El presidente de YPFB anunció que de este monto 24 millones de dólares se destinarán a tareas de explotación en el objetivo de optimizar la producción local y más de 18,3 millones de dólares a la exploración en busca de nuevas reservas de hidrocarburos.

Venezuela posee la primera reserva de gas de la región, pero se da la particularidad que no exporta gas a la región debido a la inexistencia de gasoductos o plantas regasificadoras. PDVSA redujo en el $61 \%$ su meta de inversiones para el año 2011, de acuerdo a lo previsto por el Plan Siembra Petrolera 2010-2015 que fue diseñado en 2009, tras cambiarse los plazos y las metas del proyecto original de 2005 (Tecnoil 2011). La presión fiscal desde el Ejecutivo venezolano sobre la industria petrolera se mantiene y se refleja en las previsiones de inversión y aporte social. El Plan actual engloba inversiones por 252 mil millones de dólares, de los cuales PDVSA deberá proveer 197 mil millones de dólares, para avanzar hacia cuatro objetivos estratégicos: la aceleración del desarrollo de la Faja Petrolífera del Orinoco para incorporar 2,8 millones de barriles diarios de producción para el año 2030; el desarrollo del gas Costa Afuera para satisfacer el mercado interno y exportar a mercados estratégicos; el impulso al desarrollo socialista integral del país y el equilibrio territorial; y la absoluta soberanía sobre el recurso petrolero y gasífero.

En síntesis, se observan acciones concretas sólo en algunos países de la región como Chile y Brasil, con la salvedad de que su interés es asegurar su propio abastecimiento energético, independientemente del reconocimiento de la necesidad de una estrategia supraregional para el logro de la seguridad energética que permita el desarrollo de la región. En consecuencia, los dos países con las mayores reservas de gas, pero sin inversiones en infraestructura, con inestabilidad política creciente y sin seguridad jurídica (el caso de Bolivia y Venezuela en distinto grado), están generando vulnerabilidad en países de
Geopolítica del gas en Sudamérica

Ana Lía del Valle Guerrero 
la región como Brasil y Argentina, dependientes del gas de Bolivia, pero sin ninguna certeza de abastecimiento continuo, a pesar de los contratos de largo plazo ya firmados. Argentina, por su parte, disminuyó las exportaciones a Chile e incrementó sus importaciones.

\section{Conclusiones}

El relevamiento del potencial energético de los países sudamericanos es un paso previo necesario para una explotación sustentable de los recursos que permita la utilización de todas las alternativas energéticas de que dispone la región. Recordemos que cada año que pasa es mayor la necesidad de energía y que, al no realizarse las inversiones en infraestructura necesarias en la región, el problema se hace cada vez mayor y puede generar una crisis energética regional más profunda. La vulnerabilidad es la contracara de la seguridad energética a la que aspira todo país y es la que se debe evitar con acciones conjuntas a nivel regional que favorezca la integración.

La región americana cuenta con suficiente variedad y cantidad de recursos renovables y no renovables. Víctor Bronstein, Director del Centro de Estudios de Energía, Política y Sociedad (CEEPyS) sostiene que "América Latina como región y más allá de la situación particular de cada país, se autoabastece energéticamente. Entonces, el proceso de integración es importante porque en las puertas de una nueva crisis energética global, la lucha no va a ser por el precio, sino por el recurso" (Roca 2010: 88).

En síntesis, el escenario geopolítico de la energía en la región sudamericana en 2011, centrado en el recurso gas, mostraba cambios importantes. Argentina perdió su posición como uno de los principales exportadores de gas de la región, así como también sus posibilidades de autoabastecimiento, debido a la disminución de sus reservas y falta de inversiones en tareas de exploración e infraestructura. Su dependencia actual del GNL se hace evidente si se considera que se consumieron 8 millones de metros cúbicos por día durante 2009 con un gasto estimado entre 270 y 320 millones de dólares (Clarin 2009: 25). Inclusive, se podría llegar a la paradoja de importar gas (GNL) desde Chile, su antiguo mayor consumidor. Las compras de GNL comenzaron en 2008 con 8 barcos metaneros como una salida transitoria para la temporada invernal, pero en 2010 llegaron 21 barcos y se prevé para 2011 la llegada de otros 46 barcos. Argentina ha pasado de ser un país exportador de energía a ser un país dependiente de la importación de recursos energéticos (GNL, fuel oil, combustibles). En 2010, fue una importadora neta de hidrocarburos, después de dos décadas de autoabastecimiento y, además, es vulnerable desde el punto de vista de los altos costos que debe pagar para asegurarse la provisión de esos recursos.

Brasil es el principal ganador de la región gracias a los inmensos descubrimientos de recursos petrolíferos y gasíferos realizados en su plataforma submarina durante 2007-2008 y con un plan en marcha que le permitirá la rápida 
obtención del recurso se transformaría en 2015 en un país superavitario en recursos energéticos (gas, petróleo, energía hidroeléctrica, energías alternativas). Sin embargo, a pesar de tener el autoabastecimiento petrolero desde el 2007, en el corto plazo se muestra vulnerable por su dependencia del inseguro abastecimiento de gas desde Bolivia. Al respecto, José Sergio Gabrielli, presidente de Petrobras, expresó que en 6 años los pozos de los nuevos descubrimientos en la plataforma continental brasileña entrarán en producción, con lo cual se lograría obtener en 2015 el autoabastecimiento gasífero independizándose así del inseguro abastecimiento de gas desde Bolivia.

Chile también puede considerarse un ganador, puesto que, ante la crisis de abastecimiento de gas en 2004, adoptó las medidas necesarias, como apostar al GNL e instalar plantas regasificadoras que le permiten, en 2010, estar en condiciones de autoabastecerse e inclusive exportar excedentes del recurso. Su condición es un claro ejemplo de un país dependiente pero no vulnerable, puesto que posee plantas regasificadoras y fuentes de abastecimiento seguras y variadas - aunque provengan de países alejados - que no le generan vulnerabilidad. Además, retomó la inversión en energía hidroeléctrica y carbón, así como también tomó medidas de racionamiento energético preventivo.

Venezuela, Bolivia y Ecuador son países exportadores de energía que están nacionalizando sus reservas para aumentar su margen de negociación con las grandes multinacionales con la finalidad de obtener mayores ingresos derivados de las rentas del gas y del petróleo, pero generando conflictos respecto a la seguridad jurídica de los inversores. Como consecuencia de ello, éstos no realizan las inversiones necesarias en infraestructura, lo que repercute en sus posibilidades de crecimiento. Bolivia es otro de los perdedores en la región. De ser un actor central para el abastecimiento energético regional, pasó a tener serias dificultades para cumplir con los tratados acordados con Brasil y Argentina. La nacionalización de los hidrocarburos causó que las empresas afectadas en sus intereses no realizaran las inversiones necesarias en infraestructura para garantizar una mayor producción que le permitiera, a su vez, cumplir con los volúmenes acordados en los contratos de abastecimiento.

Es un caso particular de vulnerabilidad y dependencia ya que depende, por un lado, de las inversiones en infraestructura que realicen terceros países y, por otro, de la venta de su principal recurso, el gas, a países como Argentina y Brasil, lo cual le genera vulnerabilidad por la importancia que generan estos ingresos para su PBI. Venezuela también se encuentra lejos de ser el actor principal que fue en 2008 con su propuesta del Gasoducto del Sur. La caída del precio del petróleo disminuyó sus ingresos, aunque tal vez se vea mejorada esa situación en 2011 si se mantiene el precio del petróleo por encima de los 100 dólares el barril. Además, cuestiones climáticas, como una fuerte sequía, afectaron seriamente el recurso hidroeléctrico del que depende y pusieron al descubierto sus falencias y falta de inversiones en infraestructura que eviten una dependencia del clima, más aún contando con grandes recursos en hidrocarburos. Sin embargo, el hecho de explotar principalmente el petróleo
Geopolítica del gas en Sudamérica

Ana Lía del Valle Guerrero 
le genera vulnerabilidad por la dependencia extrema del PBI del país de los recursos que obtiene de esa exportación. A pesar de ello, tiene problemas de falta de inversiones en infraestructura, al igual que Bolivia. Aunque posee las mayores reservas de gas en América, éstas aún no son explotadas y tal vez debería pensar, en el corto y mediano plazo, en la exportación a través de barcos metaneros, puesto que la idea del Gasoducto del Sur mostró muchos obstáculos que lo transforman en prácticamente inviable. Hasta el momento, Venezuela sólo le provee fuel oil a Argentina y, a pesar de ser el país de mayores reservas de gas en América, no provee gas a la región.

El ingreso de actores globales en la región, como Rusia, Francia, China, Irán y España (con inversiones en Venezuela y Argentina a través de RepsolYPF), a raíz de la presencia de abundantes recursos naturales muestra la necesidad de los consumidores de asegurarse la provisión de los mismos para no ser vulnerables y la necesidad de los poseedores de los recursos de asegurarse la posibilidad de protegerlos efectivamente, a través de un equipamiento militar disuasorio. Se busca para ello obtener la transferencia de tecnología que ofrece Francia y no EEUU, así como el apoyo de Rusia y China. Esto generó un aumento de los gastos militares en la región.

El análisis geopolítico permite comprender la crisis del gas sudamericana como producto de una realidad cambiante e incierta, e interpretar la complejidad de las dinámicas existentes en los procesos sociales, políticos y económicos que le dieron origen. También comprender las relaciones de poder que se establecen frente a la aparición de nuevas situaciones geopolíticas en el espacio regional y mundial, donde la realidad se está construyendo en la cotidianeidad. La suma de diferentes factores que se desarrollan en el trabajo permiten vislumbrar, en el corto plazo, una nueva crisis en la región en el período 2015-2020 si no se adoptan las medidas necesarias para revertir la actual situación que genera falta de seguridad energética en la región. Es aquí donde el punto de vista geopolítico y estratégico permite un análisis diferencial de los escenarios de seguridad energética que va más allá de la visión economicista predominante, ya que son decisiones políticas y no económicas las que a veces definen las acciones realizadas por los Estados. En consecuencia, promover una alianza estratégica entre Estados, abordando el tema desde una perspectiva global, sin dejar de lado las particularidades regionales, no sólo es recomendable, sino es una verdadera prioridad para Sudamérica.

\section{Bibliografía}

Barrios M. (2009), (Dir.) Diccionario latinoamericano de seguridad y geopolítica, Editorial Biblos, Buenos Aires.

Briano J. (1972), Geopolítica y geoestrategia Americana, Editorial Círculo Militar, Buenos Aires.

BP (2011), BP Statically Review of World Energy 2011. 
Cardozo, E. (2004), La gobernabilidad democrática regional y el papel (des)integrador de la energía, "Nueva Sociedad", pp.136-149.

“Clarin” (2011a), Gas de Qatar en lugar del fuel oil venezolano, 22 de enero, sección El país, p. 8.

"Clarin" (2011b), Por primera vez en 20 años el país fue importador neto de combustibles, 23 de enero, sección El país, p. 20.

"Clarin" (2011c), Venezuela frente a un año económico difícil y con menores ingresos, 23 de enero, sección El Mundo, p. 25.

"Clarin” (2011d), “El año que viene, se gastará el doble para importar gas por barco", 21 de agosto, sección El país, p. 23.

Delamer G. (2005), Estrategia Para la Política, la Empresa y la Seguridad, Instituto de Publicaciones Navales, Buenos Aires.

DoE/EIA (2007), International Energy Outlook 2007, Washington D.C.

Escribano F. G. (2006), Seguridad Energética: concepto, escenarios e implicaciones para España y la UE, "Documento de Trabajo", Real Instituto Elcano, no 33.

Escribano F. G. (2008), Entre el mercado y la geopolítica: seguridad de abastecimiento y corredores energéticos en la UE, "ICE - Economía de la Energía", no 842, mayo-junio, pp. $29-44$.

Hutschenreuter A. (2008), Las compuertas geopolíticas del siglo XX, working paper 4 -Programa geopolítica - CAEI, Centro Argentino de Estudios Internacionales, disponible en: www.caei.com.ar/es/programas/geopolitica/04.pdf

International Energy Agency, IEA (2007), World Energy Outlook 2007, París.

Isbell P. (2008), Energía y Geopolítica en América Latina, "Documento de Trabajo", no 12, Real Instituto Elcano.

Klare M. (2003), Guerras por los recursos. El futuro escenario del conflicto global, Ed. Tendencias.

Klare M. (2008), Planeta sediento, recursos menguantes. La Nueva Geopolítica de la Energía, Ediciones Urano, Barcelona.

Lindón A., Hiernaux D. (2006), (ed.), Tratado de Geografía Humana, Ed. Antrophos, México, pp. 187-199.

Marini J. (1985), El conocimiento geopolítico, Ediciones Círculo Militar, no 720, Buenos Aires.

Méndez R. (2011), El Nuevo mapa geopolítico del mundo, Ed. Tirant Lo Blanch.

"Nuevo Herald" (2011), Oppenheimer: El nuevo centro petrolero del mundo, jueves 29 de septiembre, disponible en: http://elnuevoherld.com/2011/09/28/v-print/1034152/ oppenheimer-el-nuevo-ce, consulta: 30.09.2011.

Palenzuelos E. (2008), (Dir.), El petróleo y el gas en la geoestrategia mundial, Editorial Akal, Serie Economía, Madrid.

Roca, M. (2010), Estamos a las puertas de una crisis energética global, Ed. Taeda, Buenos Aires.
Geopolítica del gas en Sudamérica

Ana Lía del Valle Guerrero 